\title{
THE LEVEL FUNCTION IN REARRANGEMENT INVARIANT SPACES
}

\author{
Gord Sinnamon
}

Abstract

An exact expression for the down norm is given in terms of the level function on all rearrangement invariant spaces and a useful approximate expression is given for the down norm on all rearrangement invariant spaces whose upper Boyd index is not one.

\section{Introduction}

Let $\lambda$ be a measure on $\mathbf{R}$ and take $X$ to be a rearrangement invariant space of $\lambda$-measurable functions. We define the down norm of a $\lambda$-measurable function $f$ to be

(1.1) $\|f\|_{X \downarrow}=\sup \left\{\int_{\mathbf{R}}|f| g d \lambda: g \geq 0, g\right.$ non-increasing, $\left.\|g\|_{X^{\prime}} \leq 1\right\}$.

Had we taken the supremum over all $g$ in the unit ball of $X^{\prime}$, the associate space of the Banach function space $X$, we would have recovered the norm of $f$ in $X$ so it is immediate that

$$
\|f\|_{X \downarrow} \leq\|f\|_{X} .
$$

The significance of the down norm is that the inequality

$$
\int_{\mathbf{R}} f g d \lambda \leq\|f\|_{X \downarrow}\|g\|_{X^{\prime}}
$$

holds for all $f$ and all non-negative, non-increasing functions $g$. Since the down norm of $f$ is smaller than the norm of $f$ in $X$ this estimate uses the monotonicity hypothesis on $g$ to improve the usual estimate

$$
\int_{\mathbf{R}} f g d \lambda \leq\|f\|_{X}\|g\|_{X^{\prime}}
$$

2000 Mathematics Subject Classification. Primary: 46E30; Secondary: 26D15.

Key words. Level function, rearrangement invariant, down norm.

Support from the Natural Sciences and Engineering Research Council of Canada is gratefully acknowledged. 
To use inequality (1.2) effectively it is necessary to understand the down norm. In the case that $X$ is Lebesgue space this has been done in two ways. Halperin $[\mathbf{6}]$ and Lorentz $[\mathbf{9}]$ gave an exact expression for the down norm when $\lambda$ is a non-negative weight function times Lebesgue measure. Given a function $f$ they constructed a related function $f^{\circ}$, called the level function of $f$, and showed that the down norm is precisely the norm of $f^{\circ}$ in $X$. In [13], the level function construction was extended to general (regular) measures on $\mathbf{R}$, the down norm was shown to define a Banach space and the dual space was also constructed.

The second approach to understanding the down norm, given in [12] for weighted Lebesgue spaces, was to give an equivalent norm in a more tractable form. The norm in $X$ of a certain averaging operator applied to $f$ was shown to be equivalent to the down norm of $f$. The loss of exactness is more than made up for because the averaging operator is linear. This approach was extended to Orlicz spaces with weights in $[\mathbf{8}]$ where the down norm in sequence spaces was also considered.

Our object is to look at both of these approaches in the more general setting of rearrangement invariant spaces. As often happens when theorems are examined in their natural generality, the proofs reduce to their essential features and greater understanding is gained. We will see how the averaging operator involved in the results of $[\mathbf{1 2}]$ and $[\mathbf{8}]$ arises naturally from the level function construction and how the finiteness of $\lambda(\mathbf{R})$ affects that operator. We will also see why the level function approach to the down norm remains valid in all rearrangement invariant spaces while a restriction is required for the other approach to be valid. In [12] the restriction was that the Lebesgue index be greater than one and in [8] a $\Delta_{2}$ condition was imposed on the $N$-functions defining the Orlicz spaces.

For definitions and notation involving Banach function spaces and rearrangement invariant spaces we refer to $[\mathbf{1}]$. We adopt the convention that $0 \cdot \infty=\infty / \infty=0$. If $A$ and $B$ are expressions involving $f$, we write $A \approx B$ to mean that there exists a positive constant $C$, not depending on $f$, such that $C^{-1} A \leq B \leq C A$. The range of integration of an integral given with limits is taken to be the closed interval so that

$$
\int_{a}^{b} f d \lambda=\int_{[a, b]} f d \lambda \text { but } \int_{-\infty}^{b} f d \lambda=\int_{(-\infty, b]} f d \lambda .
$$

\section{The down norm}

Let $X$ be a rearrangement invariant space over the measure space $(\mathbf{R}, \lambda)$. For the down norm to be interesting some restrictions on 
$\lambda$ are in order. Since we want non-negative, non-increasing functions to be $\lambda$-measurable we assume that sets of the form $(-\infty, x]$ and $(-\infty, x)$ are $\lambda$-measurable which means that all Borel sets are $\lambda$-measurable. To ensure that the space $X^{\prime}$ actually contains non-trivial non-negative, nonincreasing functions we assume that for each $x, \lambda(-\infty, x]<\infty$. For technical reasons the measure $\lambda$ in [13] was assumed to be regular and since we wish to apply those results we make the same assumption here. Finally, in working with rearrangement invariant spaces it is usual to assume that the underlying measure space is resonant [1, Definition II.2.3 and Theorem II.2.7] so that, among other things, the associate space will also be rearrangement invariant. For these reasons we assume henceforth that

$\lambda$ is a regular Borel measure on $\mathbf{R}$,

$\lambda(-\infty, x]<\infty$ for all $x \in \mathbf{R}$, and

$\lambda$ is nonatomic or else completely atomic

with all atoms having measure 1.

With these assumptions on the measure $\lambda$ we can show that $X \downarrow$, the collection of functions $f$ satisfying $\|f\|_{X \downarrow}<\infty$, is a normed vector space: It is easy to see that $X \downarrow$ is a vector space containing $X$ and it is clear from (1.1) that $\|\cdot\|_{X \downarrow}$ is non-negative, homogeneous, and satisfies the triangle inequality. It remains to show that only the zero function has zero norm in $X \downarrow$. For each $x \in \mathbf{R}, \lambda(-\infty, x]<\infty$ so $\chi_{(-\infty, x]} \in X^{\prime}$ and hence, if $\|f\|_{X \downarrow}=0$ we have $\int_{-\infty}^{x} f d \lambda=0$ for each $x$. It follows that $f=0 \lambda$-almost everywhere and we have shown that $\|\cdot\|_{X \downarrow}$ is a norm. In fact, $X \downarrow$ is a Banach space as we show in Theorem 5.3.

In most applications, the measure $\lambda$ is weighted Lebesgue measure on the half line, $d \lambda(x)=w(x) d x$ with $w$ a non-negative, locally integrable function on $[0, \infty)$. The case that $\lambda$ is counting measure on the positive integers also arises. The rearrangement invariant spaces for the latter measure include $l^{p}$ and Orlicz sequence spaces while those for the former measure are weighted Lebesgue spaces, Orlicz spaces, Lorentz spaces and others. We hasten to point out that while the weighted Lebesgue space $L_{w}^{p}[0, \infty)$ is not rearrangement invariant with respect to Lebesgue measure unless $w \equiv 1$, it is rearrangement invariant with respect to the measure $w(x) d x$.

We plan to use the level function to relate the norm in the space $X \downarrow$ to the norm on the original space $X$. The next proposition introduces the level function as constructed in $[\mathbf{1 3}]$. For convenience we define $B$ 
to be the collection of $\lambda$-measurable functions on $\mathbf{R}$ which are bounded and supported in a set of the form $(-\infty, M]$ for some $M \in \mathbf{R}$.

Proposition 2.1. Suppose $\lambda$ satisfies (2.1) and $f \in B$. Then there is a non-negative, non-increasing function $f^{\circ} \in B$, called the level function of $f$ with respect to $\lambda$, and having the following properties.

(a) There exists a finite or countable collection of disjoint intervals $I_{i}$ of finite, non-zero $\lambda$ measure such that $f=f^{\circ} \lambda$-almost everywhere on $E=\mathbf{R} \backslash \cup_{i} I_{i}$ and for each $i$,

$$
f^{\circ}(x)=\left(1 / \lambda I_{i}\right) \int_{I_{i}}|f| d \lambda
$$

for $\lambda$-almost every $x \in I_{i}$.

(b) If $g$ is non-negative and non-increasing then

$$
\int_{\mathbf{R}]}|f| g d \lambda \leq \int_{\mathbf{R}} f^{\circ} g d \lambda
$$

(c) If $f_{1}, f_{2} \in B$ and $\left|f_{1}\right| \leq\left|f_{2}\right|$ then $f_{1}^{\circ} \leq f_{2}^{\circ}$.

Proof: The structure of the level function of $f$ is given in [13, Theorem 4.4, Definition 4.6, Corollary 4.8 and Theorem 4.9]. There it is shown that $f^{\circ}$ is non-negative and non-increasing and that (a) holds. It is not assumed in $[\mathbf{1 3}]$ that $f$ is supported on $(-\infty, M]$ so the possibility of a level interval of infinite $\lambda$ measure is considered there. An easy argument shows that if $f$ is supported on $(-\infty, M]$ then all the level intervals $I_{i}$ are contained in $(-\infty, M]$ and hence are of finite $\lambda$ measure. Clearly we may discard those of zero $\lambda$-measure.

Part (b) is given in [13, Theorem 4.11] and (c) is proved in [13, Theorem 5.2].

The main result of this section is given in the next theorem for $f \in B$ and in Corollary 2.4 for general $f$.

Theorem 2.2. Suppose $\lambda$ satisfies (2.1), $X$ is a rearrangement invariant space over $(\mathbf{R}, \lambda), f \in B$, and $f^{\circ}$ is the level function of $f$ with respect to $\lambda$. Then $\|f\|_{X \downarrow}=\left\|f^{\circ}\right\|_{X}$.

Proof: We use the level intervals of $f$ to define the operator $A_{f}$.

$$
A_{f} h=h \chi_{E}+\sum_{i}\left(\frac{1}{\lambda I_{i}} \int_{I_{i}} h d \lambda\right) \chi_{I_{i}} .
$$

Note that $A_{f}$ is self-adjoint, that is $\int_{\mathbf{R}}\left(A_{f} g\right) h d \lambda=\int_{\mathbf{R}} g\left(A_{f} h\right) d \lambda$ for appropriate $g$ and $h$. Also note that by [1, Theorem II.4.8] $A_{f}$ is a contraction on any rearrangement invariant space, in particular $\left\|A_{f} h\right\|_{X^{\prime}} \leq$ 
$\|h\|_{X^{\prime}}$ for $h \in X^{\prime}$. It is clear from the definition that $A_{f}|f|=f^{\circ}$ and since the sets $I_{i}$ are intervals, $A_{f} h$ is non-negative and non-increasing whenever $h$ is.

If $g$ is non-negative and non-increasing and $\|g\|_{X^{\prime}} \leq 1$ then by Proposition 2.1(b),

$$
\int_{\mathbf{R}}|f| g d \lambda \leq \int_{\mathbf{R}} f^{\circ} g d \lambda \leq\left\|f^{\circ}\right\|_{X}
$$

so we have $\|f\|_{X \downarrow} \leq\left\|f^{\circ}\right\|_{X}$. Now we prove the reverse inequality. A simple limiting argument shows that

$$
\left\|f^{\circ}\right\|_{X}=\sup \int_{\mathbf{R}} f^{\circ} h d \lambda
$$

where the supremum is taken over non-negative functions $h \in B$ satisfying $\|h\|_{X^{\prime}} \leq 1$. For such an $h$, since $f^{\circ}$ is non-negative and nonincreasing, we have

$$
\int_{\mathbf{R}} f^{\circ} h d \lambda \leq \int_{\mathbf{R}} f^{\circ} h^{\circ} d \lambda=\int_{\mathbf{R}}\left(A_{f}|f|\right)\left(A_{h} h\right) d \lambda=\int_{\mathbf{R}}|f|\left(A_{f}\left(A_{h} h\right)\right) d \lambda .
$$

Set $g=A_{f}\left(A_{h} h\right)$. Since $A_{h} h=h^{\circ}$ is non-negative and non-increasing we see that $g$ is non-negative and non-increasing. Moreover, $\|g\|_{X^{\prime}} \leq$ $\|h\|_{X^{\prime}} \leq 1$ since both $A_{f}$ and $A_{h}$ are contractions on $X^{\prime}$. We conclude that

$\left\|f^{\circ}\right\|_{X} \leq \sup \left\{\int_{\mathbf{R}}|f| g d \lambda: g \geq 0, g\right.$ non-increasing, $\left.\|g\|_{X^{\prime}} \leq 1\right\}=\|f\|_{X \downarrow}$.

This completes the proof.

To extend the definition of the level function from $f \in B$ to all $\lambda$-measurable functions $f$ we use Proposition 2.1(c).

Definition 2.3. If $f$ is a $\lambda$-measurable function let $f_{n}=\min (|f|, n) \chi_{(-\infty, n]}$ and set $f^{\circ}=\lim _{n \rightarrow \infty} f_{n}^{\circ}$.

Clearly $f_{n} \in B$ for each $n$ so $f_{n}^{\circ}$ is defined. By Proposition 2.1(c), $\left\{f_{n}^{\circ}\right\}$ is a non-decreasing sequence so the limit in Definition 2.3 always exists as a function which takes values in $[0, \infty]$. Moreover, if $f \in B$ then $f_{n}=f$ for sufficiently large $n$ so the new definition of $f^{\circ}$ agrees with the original one.

It is immediate that, with this definition of the level function, Proposition 2.1(c) remains valid for arbitrary functions. An application of the Monotone Convergence Theorem shows that Part (b) also extends. Part (a) does not hold for arbitrary functions because the rightmost level interval may have infinite $\lambda$ measure. To what extent the structure of 
$f^{\circ}$ for an arbitrary function can be described in terms of level intervals is not clear.

The sequence $f_{n}=\min (|f|, n) \chi_{(-\infty, n]}$ in Definition 2.3 is chosen for convenience, in Section 5 we show that the definition of $f^{\circ}$ is independent of the approximating sequence.

Corollary 2.4. Suppose $\lambda$ satisfies (2.1) and $X$ is a rearrangement invariant space over $(\mathbf{R}, \lambda)$. If $f \in X \downarrow$ then $f^{\circ}$ is finite $\lambda$-almost everywhere, belongs to $X$, and $\|f\|_{X \downarrow}=\left\|f^{\circ}\right\|_{X}$.

Proof: Since $f_{n}=\min (|f|, n) \chi_{(-\infty, n]}$ is a non-decreasing sequence, Proposition 2.1(c) shows that $f_{n}^{\circ}$ is also. The Fatou property of the Banach function space $X$, Theorem 2.2, and the observation that $f_{n} \leq|f|$ show that

$$
\left\|f^{\circ}\right\|_{X}=\lim _{n \rightarrow \infty}\left\|f_{n}^{\circ}\right\|_{X}=\lim _{n \rightarrow \infty}\left\|f_{n}\right\|_{X \downarrow} \leq\|f\|_{X \downarrow} .
$$

Now [1, Lemma I.1.5(i) and Theorem I.1.4] show that $f^{\circ} \in X$ and is therefore finite $\lambda$-almost everywhere.

For each non-negative, non-increasing function $g$ with $\|g\|_{X^{\prime}} \leq 1$ we have, by the Monotone Convergence Theorem and Proposition 2.1(b),

$$
\int_{\mathbf{R}}|f| g d \lambda=\lim _{n \rightarrow \infty} \int_{\mathbf{R}} f_{n} g d \lambda \leq \lim _{n \rightarrow \infty} \int_{\mathbf{R}} f_{n}^{\circ} g d \lambda \leq \lim _{n \rightarrow \infty}\left\|f_{n}^{\circ}\right\|_{X}=\left\|f^{\circ}\right\|_{X} .
$$

Taking the supremum over all such $g$ yields $\|f\|_{X \downarrow} \leq\left\|f^{\circ}\right\|_{X}$ and completes the proof.

\section{An equivalent norm}

Expressing the down norm of a function $f$ in terms of the level function of $f$, although exact, has a major drawback. The map $f \rightarrow f^{\circ}$ is not linear, in fact, it is not even sublinear. In the Lebesgue space case it was shown in [12] that the space $X \downarrow$ has an equivalent norm which can be expressed in terms of a linear averaging operator applied to $f$. The same averaging operator was shown to work in Orlicz spaces in [8]. The linearity of this averaging operator leads to a duality principle which reduces weighted inequalities for a general operator considered over monotone functions to weighted inequalities for a modified operator considered over all functions. In this section we show that the equivalent norm and the duality principle remain valid for a wide range of rearrangement invariant spaces. Since the techniques involved are quite different this also provides new proofs of some results of [12] and [8]. 
Since $\lambda$ satisfies (2.1), its cumulative distribution function is finite on $\mathbf{R}$. Let $\Lambda(x)=\int_{-\infty}^{x} d \lambda$ for $x \in[-\infty, \infty]$ and define the averaging operator $P$ by

$$
P f(x)=\Lambda(x)^{-1} \int_{-\infty}^{x} f d \lambda+\Lambda(\infty)^{-1} \int_{-\infty}^{\infty} f d \lambda .
$$

By our convention the second term is absent, regardless of $f$, when $\Lambda(\infty)=\infty$.

Theorem 3.1. Suppose $\lambda$ satisfies (2.1) and $X$ is a rearrangement invariant space over $(\mathbf{R}, \lambda)$. Then $\|f\|_{X \downarrow} \approx\|P f\|_{X}$ for all $f \geq 0$ if and only if $P: X \rightarrow X$ is bounded.

Proof: Suppose first that $\|f\|_{X \downarrow} \approx\|P f\|_{X}$ for all $f \geq 0$. Then there exists a constant $C$ such that for any $f \in X$,

$$
\|P f\|_{X} \leq C\|f\|_{X \downarrow} \leq C\|f\|_{X}
$$

so $P: X \rightarrow X$ is bounded.

Conversely, suppose that $P: X \rightarrow X$ is bounded and hence continuous. Then there exists a constant $C$ such that $\|P f\|_{X} \leq C\|f\|_{X}$ for all $f \in X$. If $f \geq 0$ then set $f_{n}=\min (f, n) \chi_{(-\infty, n]}$ so that $f^{\circ}$ is the pointwise limit of the increasing sequence $\left\{f_{n}^{\circ}\right\}$. By Proposition 2.1(b) with $g=\chi_{(-\infty, x]}$ we have $\int_{-\infty}^{x} f_{n} d \lambda \leq \int_{-\infty}^{x} f_{n}^{\circ} d \lambda$ for each $n$ and each $x \in \mathbf{R}$. Thus

$$
\left\|P f_{n}\right\|_{X} \leq\left\|P\left(f_{n}^{\circ}\right)\right\|_{X} \leq C\left\|f_{n}^{\circ}\right\|_{X}
$$

and so, using the Monotone Convergence Theorem and the Fatou property of $X$, we have

$$
\|P f\|_{X} \leq C\left\|f^{\circ}\right\|_{X}=C\|f\|_{X \downarrow} .
$$

On the other hand, since $f_{n}^{\circ}$ is non-negative and non-increasing, $f_{n}^{\circ} \leq$ $P\left(f_{n}^{\circ}\right)$ and hence

$$
\|f\|_{X \downarrow}=\left\|f^{\circ}\right\|_{X}=\lim _{n \rightarrow \infty}\left\|f_{n}^{\circ}\right\|_{X} \leq \lim _{n \rightarrow \infty}\left\|P\left(f_{n}^{\circ}\right)\right\|_{X} .
$$

To complete the proof it will suffice to prove the following lemma since then we will have

$$
\|f\|_{X \downarrow} \leq 3 \lim _{n \rightarrow \infty}\left\|P f_{n}\right\|_{X}=3\|P f\|_{X} .
$$

Lemma 3.2. Suppose $\lambda$ satisfies (2.1) and $X$ is a rearrangement invariant space over $(\mathbf{R}, \lambda)$. Then for any non-negative $f \in B$ we have $\left\|P\left(f^{\circ}\right)\right\|_{X} \leq 3\|P f\|_{X}$ 
Proof: We show that $\left\|P\left(f^{\circ}\right)-P f\right\|_{X} \leq 2\|P f\|_{X}$, from which the result is immediate. For this argument we need a few details from [13, Definition 4.6] in addition to those presented in Proposition 2.1. With $I_{i}$ and $E$ as in Proposition 2.1, if we define $a_{i}$ and $b_{i}$ by $\left(a_{i}, b_{i}\right) \subset I_{i} \subset\left[a_{i}, b_{i}\right]$ then we have the following: The point $x \in E$ if and only if

$$
\int_{(-\infty, x)} f^{\circ} d \lambda=\int_{(-\infty, x)} f d \lambda \text { and } \int_{(-\infty, x]} f^{\circ} d \lambda=\int_{(-\infty, x]} f d \lambda .
$$

The point $x$ is interior to one of the intervals $I_{i}$ if and only if

$$
\int_{(-\infty, x)} f^{\circ} d \lambda>\int_{(-\infty, x)} f d \lambda \text { and } \int_{(-\infty, x]} f^{\circ} d \lambda>\int_{(-\infty, x]} f d \lambda .
$$

The left endpoint, $a_{i} \in I_{i}$ if and only if

$$
\int_{\left(-\infty, a_{i}\right]} f^{\circ} d \lambda>\int_{\left(-\infty, a_{i}\right]} f d \lambda
$$

The right endpoint, $b_{i} \in I_{i}$ if and only if

$$
\int_{\left(-\infty, b_{i}\right)} f^{\circ} d \lambda>\int_{\left(-\infty, b_{i}\right)} f d \lambda
$$

It follows that

$$
\begin{aligned}
P\left(f^{\circ}\right)(x) & -\operatorname{Pf}(x) \\
& =\sum_{i} \Lambda(x)^{-1}\left(\int_{-\infty}^{x} f^{\circ} d \lambda-\int_{-\infty}^{x} f d \lambda\right) \chi_{I_{i}}(x) \\
& =\sum_{i} \Lambda(x)^{-1}\left(\int_{I_{i} \cap(-\infty, x]} f^{\circ} d \lambda-\int_{I_{i} \cap(-\infty, x]} f d \lambda\right) \chi_{I_{i}}(x) \\
& \leq \sum_{i} \Lambda(x)^{-1} \int_{I_{i} \cap(-\infty, x]} f^{\circ} d \lambda \chi_{I_{i}}(x) .
\end{aligned}
$$

The second equality above is easy to prove in two cases depending on whether $a_{i} \in I_{i}$ or not.

We use Proposition 2.1(a) to continue the calculation.

$$
\begin{aligned}
P\left(f^{\circ}\right)(x) & -P f(x) \\
& \leq \sum_{i} \Lambda(x)^{-1} \int_{I_{i} \cap(-\infty, x]} d \lambda\left(\int_{I_{i}} d \lambda\right)^{-1} \int_{I_{i}} f d \lambda \chi_{I_{i}}(x) \\
& =\sum_{i} \lambda(-\infty, x]^{-1} \lambda\left(I_{i} \cap(-\infty, x]\right) \lambda\left(I_{i}\right)^{-1} \int_{I_{i}} f d \lambda \chi_{I_{i}}(x) .
\end{aligned}
$$


Now we use the obvious inequality

$$
\lambda\left(I_{i} \cap(-\infty, x]\right) \lambda\left(I_{i} \cup(-\infty, x]\right) \leq \lambda(-\infty, x] \lambda\left(I_{i}\right)
$$

to get

$$
P\left(f^{\circ}\right)(x)-P f(x) \leq \sum_{i} \lambda\left(I_{i} \cup(-\infty, x]\right)^{-1} \int_{I_{i}} f d \lambda \chi_{I_{i}}(x) .
$$

Note that for $x \in I_{i}, I_{i} \cup(-\infty, x]$ does not depend on $x$. It is either $\left(-\infty, b_{i}\right)$ or $\left(-\infty, b_{i}\right]$ depending on whether or not $b_{i}$ is in $I_{i}$. Set $B_{i}=$ $\lambda\left(I_{i} \cup(-\infty, x]\right)$.

Define $\mathcal{I}_{0}$ and $\mathcal{I}_{1}$ by

$$
\mathcal{I}_{0}=\left\{i: 2 B_{i}<\Lambda(\infty)\right\} \quad \text { and } \quad \mathcal{I}_{1}=\left\{i: 2 B_{i} \geq \Lambda(\infty)\right\}
$$

For each $i \in \mathcal{I}_{0}$ choose $c_{i} \in \mathbf{R}$ such that $\Lambda\left(c_{i}\right)=2 B_{i}$. This is possible if $\lambda$ is non-atomic because $\Lambda$ is continuous in that case. It is also possible if $\lambda$ consists of equal atoms because the condition $\lambda(-\infty, x]<\infty$ ensures that the atoms do not cluster. The reason for choosing such a $c_{i}$ is so that the set $I_{i}^{\prime}=\left(-\infty, c_{i}\right] \backslash\left(I_{i} \cup(-\infty, x]\right)$ has $\lambda$-measure $B_{i}$ for each $x \in I_{i}$.

For each $i \in \mathcal{I}_{1}$ set $I_{i}^{\prime}=I_{i}$. We claim that

$$
\left\|\sum_{i} B_{i}^{-1} \int_{I_{i}} f d \lambda \chi_{I_{i}}\right\|_{X} \leq\left\|\sum_{i} B_{i}^{-1} \int_{I_{i}} f d \lambda \chi_{I_{i}^{\prime}}\right\|_{X} .
$$

This is a familiar calculation in rearrangement invariant spaces which follows from Lemma 3.3 below. Now, if $i \in \mathcal{I}_{0}$ and $x \in I_{i}^{\prime}$ then $x \leq c_{i}$ so $2 B_{i}=\Lambda\left(c_{i}\right) \geq \Lambda(x)$. It follows that

$$
\sum_{i \in \mathcal{I}_{0}} B_{i}^{-1} \int_{I_{i}} f d \lambda \chi_{I_{i}^{\prime}}(x) \leq 2 \Lambda(x)^{-1} \sum_{i \in \mathcal{I}_{0}} \int_{I_{i}} f d \lambda \chi_{I_{i}^{\prime}}(x) \leq 2 \Lambda(x)^{-1} \int_{-\infty}^{x} f d \lambda
$$

since the intervals $I_{i}$ are disjoint. If $i \in \mathcal{I}_{1}$ then $I_{i}^{\prime}=I_{i}$ and $2 B_{i} \geq \Lambda(\infty)$ so, once again using disjointness, we have

$$
\sum_{i \in \mathcal{I}_{1}} B_{i}^{-1} \int_{I_{i}} f d \lambda \chi_{I_{i}^{\prime}}(x) \leq 2 \Lambda(\infty)^{-1} \int_{-\infty}^{\infty} f d \lambda
$$

Combining these last two estimates with (3.1) and (3.2) yields the desired inequality

$$
\left\|P\left(f^{\circ}\right)-P f\right\|_{X} \leq 2\|P f\|_{X}
$$

and completes the proof. 
Lemma 3.3. Suppose $\lambda$ satisfies (2.1), $X$ is a rearrangement invariant space over $(\mathbf{R}, \lambda)$, and for each $i$ in a countable set $\mathcal{I}$ there are subsets $I_{i}$ and $I_{i}^{\prime}$ of $\mathbf{R}$ satisfying $\lambda\left(I_{i}\right)=\lambda\left(I_{i}^{\prime}\right)<\infty$. If the sets $I_{i}$ are pairwise disjoint then

$$
\left\|\sum_{i} A_{i} \chi_{I_{i}}\right\|_{X} \leq\left\|\sum_{i} A_{i} \chi_{I_{i}^{\prime}}\right\|_{X}
$$

for all $A_{i} \geq 0$.

Proof: There is no loss of generality in assuming that all the $A_{i}$ are positive and that each $I_{i}$ has positive $\lambda$-measure. First suppose that the index set $\mathcal{I}$ is finite, having $n$ elements. In this case we may re-order if necessary so that $A_{1} \geq A_{2} \geq \cdots \geq A_{n}>0$. Let

$$
f=\sum_{i=1}^{n} A_{i} \chi_{I_{i}}, \quad g=\sum_{i=1}^{n} A_{i} \chi_{I_{i}^{\prime}}
$$

and define $t_{0}, t_{1}, \ldots, t_{n}$ by

$$
t_{0}=0 ; \quad t_{j}=\sum_{i=1}^{j} \lambda\left(I_{i}\right)=\sum_{i=1}^{j} \lambda\left(I_{i}^{\prime}\right), \quad j=1, \ldots, n .
$$

Note that $t_{j}>t_{j-1}$ for each $j$. As in [1, Example II.1.6a] we have

$$
f^{*}=\sum_{i=1}^{n} A_{i} \chi_{\left[t_{i-1}, t_{i}\right)} .
$$

Here $f^{*}$ denotes the non-increasing rearrangement of $f$ with respect to $\lambda,[\mathbf{1}$, Definition II.1.5].

Fix $j$ and set $g_{j}=\sum_{i=1}^{j} A_{i} \chi_{I_{i}^{\prime}}$. Since the $A_{i}$ are positive, $g_{j} \leq g$ and hence $g_{j}^{*} \leq g^{*}$ by [1, Proposition II.1.7]. The formula for $f^{*}$ yields

$$
\int_{0}^{t_{j}} f^{*}=\sum_{i=1}^{j} A_{i}\left(t_{i}-t_{i-1}\right)=\sum_{i=1}^{j} A_{i} \lambda\left(I_{i}^{\prime}\right)=\int_{\mathbf{R}} g_{j} d \lambda .
$$

Now $g_{j}$ and $g_{j}^{*}$ are equimeasurable and $g_{j}$ is supported in a set whose $\lambda$ measure is no greater than $t_{j}$ so we have

$$
\int_{\mathbf{R}} g_{j} d \lambda=\int_{0}^{t_{j}} g_{j}^{*} \leq \int_{0}^{t_{j}} g^{*}
$$


Thus $\int_{0}^{t_{j}} f^{*} \leq \int_{0}^{t_{j}} g^{*}$ for each $j$. If $0<t \leq t_{n}$ then we can choose $j$ so that $t_{j-1}<t \leq t_{j}$. For this $j$, using the formula for $f^{*}$ to exploit the fact that $f^{*}$ is constant on $\left(t_{j-1}, t_{j}\right)$, we get

$$
\begin{aligned}
\int_{0}^{t} f^{*} & =\frac{t_{j}-t}{t_{j}-t_{j-1}} \int_{0}^{t_{j-1}} f^{*}+\frac{t-t_{j-1}}{t_{j}-t_{j-1}} \int_{0}^{t_{j}} f^{*} \\
& \leq \frac{t_{j}-t}{t_{j}-t_{j-1}} \int_{0}^{t_{j-1}} g^{*}+\frac{t-t_{j-1}}{t_{j}-t_{j-1}} \int_{0}^{t_{j}} g^{*} \leq \int_{0}^{t} g^{*}
\end{aligned}
$$

where the last inequality is justified because $g^{*}$ is non-increasing and hence $\int_{0}^{t} g^{*}$ is a concave function. Both $f^{*}$ and $g^{*}$ vanish on $\left(t_{n}, \infty\right)$ so, for $t>t_{n}, \int_{0}^{t} f^{*}=\int_{0}^{t_{n}} f^{*} \leq \int_{0}^{t_{n}} g^{*}=\int_{0}^{t} g^{*}$, and we have established

$$
\int_{0}^{t} f^{*} \leq \int_{0}^{t} g^{*}
$$

for all $t>0$. By [1, Corollary II.4.7] this implies that $\|f\|_{X} \leq\|g\|_{X}$, which completes the proof in the finite index set case. The case of infinite $\mathcal{I}$ follows easily using the Fatou property of the space $X$.

\section{Boyd indices}

The purpose of this section is to show that the equivalent norm on the space $X \downarrow$, given in Theorem 3.1, is valid for a large class of rearrangement invariant spaces. Specifically, we show that it holds precisely when the upper Boyd index of the space is not 1. For a definition of the Boyd indices of a rearrangement invariant space which includes the case that $\lambda$ is purely atomic see $[\mathbf{2}]$. We follow [1] in denoting the upper Boyd index of $X$ by $\bar{\alpha}_{X}$. Note that for every space $X, 0 \leq \bar{\alpha}_{X} \leq 1$.

We relate the Boyd index of $X$ to the boundedness of $P$ on $X$ in two cases, when the measure $\lambda$ is purely atomic and when it is non-atomic.

Lemma 4.1. Suppose that $\lambda$ is a purely atomic measure satisfying (2.1) and $X$ is a rearrangement invariant space over $(\mathbf{R}, \lambda)$. Then $P: X \rightarrow X$ if and only if $\bar{\alpha}_{X}<1$.

Proof: We begin by applying the Luxemburg representation theorem, [13, Theorem 4.10] to assert the existence of a rearrangement invariant space $\bar{X}$ over $\mathbf{R}^{+}$, the half line with Lebesgue measure, such that

$$
\|f\|_{X}=\left\|f^{*}\right\|_{\bar{X}}
$$

for all $f \in X$. 
In the proof of $\left[\mathbf{2}\right.$, Theorem 1], D. Boyd shows that $\bar{\alpha}_{X}<1$ if and only if there exists a $C>0$ such that

$$
\left\|P_{1} f^{*}\right\|_{\bar{X}} \leq C\left\|f^{*}\right\|_{\bar{X}}, \quad f \in X
$$

Here $P_{1} \varphi$ is the step function whose value on $(k-1, k)$ is $\frac{1}{k} \int_{0}^{k} \varphi$. It remains to show that (4.1) is equivalent to $P: X \rightarrow X$.

Let $a_{1}, a_{2}, a_{3}, \ldots$ be the atoms of $\lambda$ arranged in increasing order. This is possible since the hypothesis $\lambda(-\infty, x]<\infty$ ensures that there are at most finitely many atoms to the left of any given real number.

Suppose first that (4.1) holds for some $C>0$ and fix $f \in X$. Define the sequence $\left\{f_{j}\right\}$ by $f_{j}=\left|f\left(a_{j}\right)\right|$ and let $\left\{f_{j}^{*}\right\}$ be the same sequence arranged in non-increasing order. The non-increasing rearrangement $f^{*}$ of $f$ is a step function whose value on $(j-1, j)$ is $f_{j}^{*}$ for $j=1,2, \ldots$. Thus the value of $P_{1} f^{*}$ on $(k-1, k)$ is $\frac{1}{k} \sum_{j=1}^{k} f_{j}^{*}$.

Since $\Lambda\left(a_{k}\right)$ is the number of atoms of $\lambda$ which are less than or equal to $a_{k}$ and the $a_{j}$ 's are in order we have $\Lambda\left(a_{k}\right)=k$. Therefore

$$
\operatorname{Pf}\left(a_{k}\right) \leq \Lambda\left(a_{k}\right)^{-1} \int_{-\infty}^{a_{k}}|f| d \lambda=\frac{1}{k} \sum_{j=1}^{k} f_{j} \leq \frac{1}{k} \sum_{j=1}^{k} f_{j}^{*} .
$$

Since the sequence $\left\{\frac{1}{k} \sum_{j=1}^{k} f_{j}^{*}\right\}$ is already in non-increasing order, if we arrange the terms of the sequence $\left\{P f\left(a_{k}\right)\right\}$ in non-increasing order the result will remain termwise less than or equal to $\left\{\frac{1}{k} \sum_{j=1}^{k} f_{j}^{*}\right\}$. Thus $(P f)^{*}$ is a step function whose value on $(k-1, k)$ is less than or equal to $\frac{1}{k} \sum_{j=1}^{k} f_{j}^{*}$. We conclude that $(P f)^{*} \leq P_{1} f^{*}$ and we obtain

$$
\|P f\|_{X}=\left\|(P f)^{*}\right\|_{\bar{X}} \leq\left\|P_{1} f^{*}\right\|_{\bar{X}} \leq C\left\|f^{*}\right\|_{\bar{X}}=C\|f\|_{X}
$$

so $P: X \rightarrow X$.

To prove the converse suppose that $P: X \rightarrow X$, that is, suppose that there exists a constant $C$ such that $\|P g\|_{X} \leq C\|g\|_{X}$ for all $g \in X$. Fix $f \in X$, define $\left\{f_{j}\right\}$ and $\left\{f_{j}^{*}\right\}$ as above, and define $g$ by $g\left(a_{j}\right)=f_{j}^{*}$. This defines $g$ on a set of full $\lambda$ measure and since evidently $f^{*}=g^{*}$ we see that $g \in X$. Now $P g$ is a non-increasing function so $(P g)^{*}$ is a step function whose value on $(k-1, k)$ is $P g\left(a_{k}\right)=\frac{1}{k} \sum_{j=1}^{k} g\left(a_{j}\right)=\frac{1}{k} \sum_{j=1}^{k} f_{j}^{*}$. That is, $(P g)^{*}=P_{1} f^{*}$. We have

$$
\left\|P_{1} f^{*}\right\|_{\bar{X}}=\left\|(P g)^{*}\right\|_{\bar{X}}=\|P g\|_{X} \leq C\|g\|_{X}=C\left\|g^{*}\right\|_{\bar{X}}=C\left\|f^{*}\right\|_{\bar{X}} .
$$

This completes the proof. 
Lemma 4.2. Suppose that $\lambda$ is a non-atomic measure satisfying (2.1) and $\varphi$ is a non-increasing, right continuous function. Then

$$
(\varphi \circ \Lambda)^{*}=\varphi \chi_{[0, \Lambda(\infty))} .
$$

Here $\Lambda(x)=\int_{-\infty}^{x} d \lambda$.

Proof: Since the right hand side of (4.2) is non-increasing and right continuous it is enough to show that it is equimeasurable with $\varphi \circ \Lambda$, that is, for each $\gamma>0$

$$
\lambda\{x \in \mathbf{R}: \varphi \circ \Lambda(x)>\gamma\}=|\{t \in[0, \Lambda(\infty)): \varphi(t)>\gamma\}| .
$$

The right hand side is the Lebesgue measure of a set of the form $[0, s)$ for some $s \in[0, \Lambda(\infty)]$ so we wish to show that

$$
\lambda\left(\Lambda^{-1}([0, s))\right)=s, \quad s \in[0, \Lambda(\infty)] .
$$

It is clear that (4.3) holds for $s=0$ and $s=\Lambda(\infty)$ and since $\lambda$ is nonatomic, every $s \in(0, \Lambda(\infty))$ may be expressed as $s=\Lambda(y)$ for some $y \in \mathbf{R}$.

Since $(-\infty, y] \subset \Lambda^{-1}([0, \Lambda(y)])$ we have $\Lambda(y) \leq \lambda\left(\Lambda^{-1}([0, \Lambda(y)])\right.$ and since $\Lambda^{-1}([0, \Lambda(y)])=\sup _{\Lambda(x) \leq \Lambda(y)}(-\infty, x]$ we have

$$
\lambda\left(\Lambda^{-1}([0, \Lambda(y)]) \leq \sup _{\Lambda(x) \leq \Lambda(y)} \Lambda(x) \leq \Lambda(y) .\right.
$$

It follows that $\lambda\left(\Lambda^{-1}([0, s])\right)=s$. It is easy to see that $\lambda\left(\Lambda^{-1}(\{s\})\right)=0$ for all $s$ so we have (4.3) as required. This completes the proof.

Lemma 4.3. If $\lambda$ is a non-atomic measure satisfying (2.1) then $P: X \rightarrow$ $X$ if and only if $\bar{\alpha}_{X}<1$.

Proof: We begin by applying the Luxemburg representation theorem, [13, Theorem 4.10] to assert the existence of a rearrangement invariant space $\bar{X}$ over $\mathbf{R}^{+}$, the half line with Lebesgue measure, such that

$$
\|f\|_{X}=\left\|f^{*}\right\|_{\bar{X}}
$$

for all $f \in X$.

In the proof of [2, Theorem 1], D. Boyd shows that $\bar{\alpha}_{X}<1$ if and only if there exists a $C>0$ such that

$$
\left\|P_{1} f^{*}\right\|_{\bar{X}} \leq C\left\|f^{*}\right\|_{\bar{X}}, \quad f \in X
$$

Here $P_{1}$ is defined by $P_{1} \varphi(t)=\chi_{[0, \Lambda(\infty))}(t) t^{-1} \int_{0}^{t} \varphi$. It remains to show that (4.4) is equivalent to $P: X \rightarrow X$. 
Define the operator $p$ by

$$
p f(x)=\Lambda(x)^{-1} \int_{-\infty}^{x} f d \lambda
$$

so that $P f(x)=p f(x)+p f(\infty)$. Note that if $\lambda$ is an infinite measure then $P=p$. If $\lambda$ is a finite measure then the second term in $P$ is always bounded on $X$ because by duality we have

$$
\|p f(\infty)\|_{X}=\|1\|_{X}\left|\Lambda(\infty)^{-1} \int_{-\infty}^{\infty} f d \lambda\right| \leq \Lambda(\infty)^{-1}\|1\|_{X}\|1\|_{X^{\prime}}\|f\|_{X} .
$$

Over a finite measure space the constant function 1 is in every rearrangement invariant space. This shows that $P: X \rightarrow X$ if and only if $p: X \rightarrow X$ so from now on we restrict our attention to the operator $p$.

Fix $y \in \mathbf{R}$ and choose $y_{1}$ minimal such that $\Lambda\left(y_{1}\right)=\Lambda(y)$. Since $\lambda$ is non-atomic and $\lambda\left(y_{1}, y\right]=0$, the functions $\chi_{(-\infty, y)}$ and $\chi_{\left(-\infty, y_{1}\right)}$ agree $\lambda$-almost everywhere. The choice of $y_{1}$ ensures that $\chi_{\left(-\infty, y_{1}\right)}=$ $\chi_{[0, \Lambda(y))} \circ \Lambda$ so we have

$$
\left(\chi_{(-\infty, y)}\right)^{*}=\left(\chi_{\left(-\infty, y_{1}\right)}\right)^{*}=\left(\chi_{[0, \Lambda(y))} \circ \Lambda\right)^{*}=\chi_{[0, \Lambda(y))}
$$

by Lemma 4.2. Now we use [1, Theorem II.2.2] to get

$$
\int_{-\infty}^{y} f d \lambda=\int_{-\infty}^{\infty} f \chi_{(-\infty, y)} d \lambda \leq \int_{0}^{\infty} f^{*}\left(\chi_{(-\infty, y)}\right)^{*}=\int_{0}^{\Lambda(y)} f^{*} .
$$

That is, $\Lambda(y) p f(y) \leq \Lambda(y) P_{1}\left(f^{*}\right)(\Lambda(y))$. Since this holds for all $y \in \mathbf{R}$ we have $p f \leq P_{1}\left(f^{*}\right) \circ \Lambda$. Now we use [1, Proposition II.1.7] and apply Lemma 4.2 for a second time to get

$$
(p f)^{*} \leq\left(\left(P_{1} f^{*}\right) \circ \Lambda\right)^{*}=P_{1}\left(f^{*}\right) .
$$

Suppose now that (4.4) holds. For each $f \in X$

$$
\|p f\|_{X}=\left\|(p f)^{*}\right\|_{\bar{X}} \leq\left\|P_{1} f^{*}\right\|_{\bar{X}} \leq C\left\|f^{*}\right\|_{\bar{X}}=C\|f\|_{X} .
$$

Thus $p: X \rightarrow X$.

It is an easy exercise to show that $\{x \in \mathbf{R}: \Lambda(x)<\Lambda(y)\}=(-\infty, y)$ for $\lambda$-almost every $y \in \mathbf{R}$. For such a $y$ and a fixed $f \in X$ set $\varphi=$ $f^{*} \chi_{[0, \Lambda(y))}$. Applying Lemma 4.2 once again we have

$$
\begin{aligned}
\Lambda(y)\left(P_{1} f^{*}\right) \circ \Lambda(y) & =\int_{0}^{\Lambda(y)} f^{*}=\int_{0}^{\Lambda(\infty)} \varphi=\int_{0}^{\infty}(\varphi \circ \Lambda)^{*} \\
& =\int_{-\infty}^{\infty} \varphi \circ \Lambda d \lambda=\int_{-\infty}^{y} f^{*} \circ \Lambda d \lambda=\Lambda(y) p\left(f^{*} \circ \Lambda\right)(y) .
\end{aligned}
$$


Thus $\left(P_{1} f^{*}\right) \circ \Lambda$ and $p\left(f^{*} \circ \Lambda\right)$ are equal, considered as $\lambda$-measurable functions.

Now suppose that $p: X \rightarrow X$ is bounded, that is, suppose that there exists a constant $C$ such that $\|p f\|_{X} \leq C\|f\|_{X}$ for all $f \in X$. Fix $f \in X$. Both $f^{*}$ and $P_{1} f^{*}$ are non-increasing and right continuous so we may apply Lemma 4.2 twice to get $\left(f^{*} \circ \Lambda\right)^{*}=f^{*} \chi_{[0, \Lambda(\infty))}$ and $\left(\left(P_{1} f^{*}\right) \circ \Lambda\right)^{*}=P_{1} f^{*} \chi_{[0, \Lambda(\infty))}$. Since both $f^{*}$ and $P_{1} f^{*}$ are supported in $[0, \Lambda(\infty))$ we have $\left(f^{*} \circ \Lambda\right)^{*}=f^{*}$ and $\left(\left(P_{1} f^{*}\right) \circ \Lambda\right)^{*}=P_{1} f^{*}$.

With these observations we get

$$
\begin{aligned}
\left\|P_{1} f^{*}\right\|_{\bar{X}}=\left\|\left(\left(P_{1} f^{*}\right) \circ \Lambda\right)^{*}\right\|_{\bar{X}}=\left\|\left(P_{1} f^{*}\right) \circ \Lambda\right\|_{X}=\left\|p\left(f^{*} \circ \Lambda\right)\right\|_{X} \\
\leq C\left\|f^{*} \circ \Lambda\right\|_{X}=C\left\|\left(f^{*} \circ \Lambda\right)^{*}\right\|_{\bar{X}}=C\left\|f^{*}\right\|_{\bar{X}} .
\end{aligned}
$$

This shows that (4.4) holds and completes the proof.

Combining Corollary 2.4, Theorem 3.1, Lemma 4.1, and Lemma 4.3 yields

Theorem 4.4. Suppose $\lambda$ satisfies (2.1) and $X$ is a rearrangement invariant space over $(\mathbf{R}, \lambda)$. Then $\|f\|_{X \downarrow}=\left\|f^{\circ}\right\|_{X}$ and the following are equivalent

(a) $\|f\|_{X \downarrow} \approx\|P f\|_{X}$ for $f \in X \downarrow$

(b) $P: X \rightarrow X$ is a bounded operator, and

(c) $\bar{\alpha}_{X}<1$.

We now present the duality principle mentioned earlier.

Theorem 4.5. Suppose $\lambda$ satisfies (2.1) and $X$ is a rearrangement invariant space over $(\mathbf{R}, \lambda)$ such that $\bar{\alpha}_{X}<1$. Suppose also that $Y$ is a Banach function space over the measure space $(M, \mu)$ and that $T$ and $T^{*}$ are operators on $\mu$ and $\lambda$ measurable functions respectively such that

$$
\int_{\mathbf{R}}(T f) g d \lambda=\int_{M} f\left(T^{*} g\right) d \mu
$$

for all $f \in Y$ and all non-negative, non-increasing $g \in X^{\prime}$. Then there exists a constant $C_{1}$ such that

$$
\|P T f\|_{X} \leq C_{1}\|f\|_{Y}
$$

for all $f \in Y$ if and only if there exists a constant $C_{2}$ such that

$$
\left\|T^{*} g\right\|_{Y^{\prime}} \leq C_{2}\|g\|_{X^{\prime}}
$$

for all non-negative, non-increasing $g \in X^{\prime}$. 
Proof: Since $\bar{\alpha}_{X}<1$, Theorem 4.4 shows that there exists a positive constant $C$ such that

$$
C^{-1}\|P \varphi\|_{X} \leq\|\varphi\|_{X \downarrow} \leq C\|P \varphi\|_{X}
$$

for all $\varphi \in X \downarrow$.

If a $C_{1}$ exists satisfying (4.5) then for any $f \in Y$ and any non-negative, non-increasing $g \in X^{\prime}$ we have

$$
\begin{aligned}
\int_{M} f\left(T^{*} g\right) d \mu=\int_{\mathbf{R}}(T f) g d \lambda & \leq\|T f\|_{X \downarrow}\|g\|_{X^{\prime}} \\
& \leq C\|P T f\|_{X}\|g\|_{X^{\prime}} \leq C C_{1}\|f\|_{Y}\|g\|_{X^{\prime}}
\end{aligned}
$$

Taking the supremum over all $f$ with $\|f\|_{Y} \leq 1$ yields (4.6) with $C_{2}=$ $C C_{1}$.

Conversely, if there exists a $C_{2}$ satisfying (4.6) then for any $f \in Y$ and any non-negative, non-increasing $g \in X^{\prime}$ we have

$$
\int_{\mathbf{R}}(T f) g d \lambda=\int_{M} f\left(T^{*} g\right) d \mu \leq\|f\|_{Y}\left\|T^{*} g\right\|_{Y^{\prime}} \leq C_{2}\|f\|_{Y}\|g\|_{X^{\prime}} .
$$

Taking the supremum over all non-negative, non-increasing $g \in X^{\prime}$ with $\|g\|_{X^{\prime}} \leq 1$ we have $\|T f\|_{X \downarrow} \leq C_{2}\|f\|_{Y}$ and hence

$$
\|P T f\|_{X} \leq C\|T f\|_{X \downarrow} \leq C C_{2}\|f\|_{Y}
$$

and so (4.5) holds with $C_{1}=C C_{2}$.

The Boyd indices are known for many classes of rearrangement invariant spaces. The simplest is the class of Lebesgue spaces. For $1 \leq p \leq$ $\infty$ let $L_{\lambda}^{p}$ denote the collection of $\lambda$-measurable functions $f$ such that $\|f\|_{L_{\lambda}^{p}}<\infty$ where

$$
\|f\|_{L_{\lambda}^{p}} \equiv\left(\int_{\mathbf{R}}|f|^{p} d \lambda\right)^{1 / p} \text { for } p<\infty \text { and }\|f\|_{L_{\lambda}^{\infty}} \equiv \underset{x \in \mathbf{R}}{\operatorname{ess} \sup _{\lambda}|f(x)| .}
$$

It is well known that the upper Boyd index of $L_{\lambda}^{p}$ is $1 / p$. Theorem 4.4 reduces to the following.

Proposition 4.6. Suppose $\lambda$ satisfies (2.1) and $1 \leq p \leq \infty$. Then $P: L_{\lambda}^{p} \rightarrow L_{\lambda}^{p}$ if and only if $1<p \leq \infty$ if and only if

$$
\begin{aligned}
\sup \left\{\int_{\mathbf{R}} f g d \lambda: g \geq 0, g \text { non-increasing, }\|g\|_{L_{\lambda}^{p^{\prime}}}\right. & \leq 1\} \\
& \equiv\|f\|_{L_{\lambda}^{p} \downarrow} \approx\|P f\|_{L_{\lambda}^{p}} .
\end{aligned}
$$


Note that since $\lambda$ may be counting measure on the set of positive integers this includes the case $L_{\lambda}^{p}=l^{p}$.

If $1<p<\infty$ and $v$ is a non-negative weight defined on $(0, \infty)$ then we may define $\lambda$ by $d \lambda(x)=\chi_{(0, \infty)}(x) v(x) d x$ and replace $f$ by $f / v$ to obtain

$$
\begin{aligned}
& \sup \left\{\int_{0}^{\infty} f g: g \geq 0, g \text { non-increasing, }\|g\|_{L_{v}^{p^{\prime}}} \leq 1\right\} \\
& \approx\left(\int_{0}^{\infty}\left(\int_{0}^{x} f\right)^{p}\left(\int_{0}^{x} v\right)^{-p} v(x) d x\right)^{1 / p}+\left(\int_{0}^{\infty} f\right)\left(\int_{0}^{\infty} v\right)^{-1 / p^{\prime}}
\end{aligned}
$$

which was proved in $[\mathbf{1 2}$, Theorem 1].

Considerable progress has been made on determining the Boyd indices of Orlicz spaces in $[\mathbf{3}],[\mathbf{4}],[\mathbf{5}],[\mathbf{1 0}]$ and others but only a small portion of this theory is required for our purposes. We refer to [11] for the definitions of a Young's function $\Phi$, its complementary Young's function $\Psi$, and the Orlicz space $L_{\lambda}^{\Phi}$. We say a Young's function satisfies the $\Delta_{2}$ condition and write $\Phi \in \Delta_{2}$ provided there exists a constant $C>1$ such that $\Phi(2 x) \leq C \Phi(x)$ for all $x>0$. We say that $\Phi$ satisfies the $\Delta_{2}^{\infty}$ condition and write $\Phi \in \Delta_{2}^{\infty}$ provided there exist constants $N>0$ and $C>1$ such that $\Phi(2 x) \leq C \Phi(x)$ for all $x>N$.

Proposition 4.7. Suppose $\lambda$ satisfies (2.1), $\Phi$ is a Young's function, and $\Psi$ is its complementary Young's function. Then $P: L_{\lambda}^{\Phi} \rightarrow L_{\lambda}^{\Phi}$ if and only if $\Psi \in \Delta_{2}^{\infty}$ if and only if

$$
\begin{aligned}
\sup \left\{\int_{\mathbf{R}} f g d \lambda: g \geq 0, g\right. \text { non-increasing, } & \left.\|g\|_{L_{\lambda}^{\Psi}} \leq 1\right\} \\
& \equiv\|f\|_{L_{\lambda}^{\Phi} \downarrow} \approx\|P f\|_{L_{\lambda}^{\Phi}} .
\end{aligned}
$$

Proof: The associate space of $L_{\lambda}^{\Phi}$ is $L_{\lambda}^{\Psi}$ with equivalent norms so all that is needed to deduce this result from Theorem 4.4 is to verify that the upper Boyd index of $L_{\lambda}^{\Phi}$ is less than one if and only if $\Psi \in \Delta_{2}^{\infty}$. Since the upper Boyd index of $L_{\lambda}^{\Phi}$ is one minus the lower Boyd index of $L_{\lambda}^{\Psi}$ we wish to show that the lower Boyd index of $\Psi$ is greater than zero if and only if $\Psi \in \Delta_{2}^{\infty}$. This follows from [10, Theorem $3.2 \mathrm{~b}$ and Theorem 4.2].

When $\lambda$ is weighted Lebesgue measure on the half line, or $\lambda$ is counting measure on the positive integers (4.7) was established in $[\mathbf{8}$, Theorem 2.2 and Theorem 3.2] under the assumption that both $\Phi$ and $\Psi$ satisfy the $\Delta_{2}$ condition. For sequence spaces Heinig and Kufner give somewhat 
more. Their Theorem 3.2 includes a weighted version of the down norm which suggests the following problem.

Problem 4.8. Suppose $\lambda$ satisfies $(2.1), X$ is a rearrangement invariant space over $(\mathbf{R}, \lambda)$, and $v$ is a non-negative, $\lambda$-measurable function. Characterize the norm

$$
\|f\|_{X_{v}^{\downarrow}}=\sup \left\{\int_{\mathbf{R}}|f| g d \lambda: g \geq 0, g \text { non-increasing, }\|g v\|_{X^{\prime}} \leq 1\right\} \text {. }
$$

\section{Completeness and duality}

We have seen that $X \downarrow$ is a normed vector space. In this section we show that $X \downarrow$ is a Banach space of functions which is not, in general, a Banach function space. We also characterize the dual space of $X \downarrow$. To begin we show that the map $f \rightarrow f^{\circ}$ preserves increasing limits.

Proposition 5.1. Suppose that $\lambda$ satisfies (2.1) and $f \in B$. If $0 \leq f_{n} \uparrow$ $|f|$ then $f_{n}^{\circ} \uparrow f^{\circ}$.

Proof: Since $f \in B, f_{n} \in B$ for all $n$ and hence $f, f_{n} \in L_{\lambda}^{2} \subset L_{\lambda \downarrow}^{2} \downarrow$ for all $n$. By [13, Theorem 5.4] $f^{\circ}$ is the unique 2-level function of $f$ and $f_{n}^{\circ}$ is the unique 2-level function of $f_{n}$. Now [13, Lemma 5.3] with $h_{n}=f_{n}^{\circ}$ shows that $\lim _{n \rightarrow \infty} f_{n}^{\circ}$ is also a 2-level function of $f$. We conclude that $\lim _{n \rightarrow \infty} f_{n}^{\circ}=f^{\circ}$ as required.

Theorem 5.2. Suppose that $\lambda$ satisfies (2.1) and $X$ is a rearrangement invariant space over $(\mathbf{R}, \lambda)$. If $0 \leq f_{n} \uparrow|f|$ then $f_{n}^{\circ} \uparrow f^{\circ}$ and $\left\|f_{n}\right\|_{X \downarrow} \uparrow$ $\|f\|_{X \downarrow}$.

Proof: First note that Proposition 2.1(c) easily extends to arbitrary functions and therefore $f_{n} \leq|f|$ implies $f_{n}^{\circ} \leq f^{\circ}$ and we have $\lim _{n \rightarrow \infty} f_{n}^{\circ} \leq$ $f^{\circ}$.

To prove the other inequality let $h=|f|$, set $h_{n}=\min (h, n) \chi_{(-\infty, n]}$ and define

$$
m_{n, k}=\min \left(f_{n}, h_{k}\right) .
$$

Since $f_{n} \uparrow h \geq h_{k}$ for all $k$, we have $\lim _{n \rightarrow \infty} m_{n, k}=h_{k}$ for all $k$. Since $h_{k} \in B$, Proposition 5.1 shows that $\lim _{n \rightarrow \infty} m_{n, k}^{\circ}=h_{k}^{\circ}$ for all $k$. Now by Definition 2.3

$$
f^{\circ}=\lim _{k \rightarrow \infty} h_{k}^{\circ}=\lim _{k \rightarrow \infty} \lim _{n \rightarrow \infty} m_{n, k}^{\circ} \leq \lim _{k \rightarrow \infty} \lim _{n \rightarrow \infty} f_{n}^{\circ}=\lim _{n \rightarrow \infty} f_{n}^{\circ} .
$$


Thus we have $f_{n}^{\circ} \uparrow f^{\circ}$. Now we apply Corollary 2.4 and the Fatou property in $X$ to get

$$
\lim _{n \rightarrow \infty}\left\|f_{n}\right\|_{X \downarrow}=\lim _{n \rightarrow \infty}\left\|f_{n}^{\circ}\right\|_{X}=\left\|f^{\circ}\right\|_{X}=\|f\|_{X \downarrow} .
$$

This completes the proof.

Theorem 5.3. If $\lambda$ satisfies (2.1) and $X$ is a rearrangement invariant space over $(\mathbf{R}, \lambda)$ then $X \downarrow$ is a Banach space.

Proof: We have already shown that $X \downarrow$ is a normed linear space, it remains to prove completeness. To do this we show that every absolutely summable sequence in $X \downarrow$ is summable in $X \downarrow$. Suppose that $f_{n} \in X \downarrow$ for all $n$ and $\sum_{n=1}^{\infty}\left\|f_{n}\right\|_{X \downarrow}<\infty$. Then $\left|f_{n}\right| \in X \downarrow$ and so $S_{N} \equiv \sum_{n=1}^{N}\left|f_{n}\right| \in X \downarrow$ for each $N$. Let $S$ be the pointwise limit of the non-decreasing sequence $S_{N}$, that is, $S=\sum_{n=1}^{\infty}\left|f_{n}\right|$. Since $S_{N} \uparrow S$ and

$$
\lim _{N \rightarrow \infty}\left\|S_{N}\right\|_{X \downarrow} \leq \sum_{n=1}^{N}\left\|f_{n}\right\|_{X \downarrow} \leq \sum_{n=1}^{\infty}\left\|f_{n}\right\|_{X \downarrow}<\infty
$$

we have $\|S\|_{X \downarrow}<\infty$ by Theorem 5.2 and hence $S \in X \downarrow$. In particular this implies that $S$ is finite $\lambda$-almost everywhere because for any $M \in \mathbf{R}$, $\chi_{(-\infty, M]}$ is non-increasing so

$$
\int_{-\infty}^{M} S d \lambda \leq\|S\|_{X \downarrow}\left\|\chi_{(-\infty, M]}\right\|_{X^{\prime}}<\infty .
$$

(Since $\lambda(-\infty, M]$ is finite, $\chi_{(-\infty, M]} \in X^{\prime}$.) Thus, $S$ is finite $\lambda$-almost everywhere on $(-\infty, M]$ but since $M$ was arbitrary, $S$ is finite $\lambda$-almost everywhere on $\mathbf{R}$.

We have shown that $\sum_{n=1}^{\infty}\left|f_{n}\right|$ converges pointwise $\lambda$-almost everywhere and it follows that $\sum_{n=1}^{\infty} f_{n}$ converges pointwise $\lambda$-almost everywhere. Let $F_{N}=\sum_{n=1}^{N} f_{n}$ and $F=\sum_{n=1}^{\infty} f_{n}$. Fix $K$, set $I_{N}=$ $\inf _{n \geq N}\left|F_{n}-F_{K}\right| \leq\left|F_{N}-F_{K}\right|$ for $N>K$ and note that $I_{N} \in X \downarrow$ with $\left\|I_{N}\right\|_{X \downarrow} \leq \sum_{n=K+1}^{N}\left\|f_{n}\right\|_{X \downarrow} \leq \sum_{n=K+1}^{\infty}\left\|f_{n}\right\|_{X \downarrow}$. The sequence $I_{N}$ is non-decreasing and converges pointwise to $\left|F-F_{K}\right|$. Thus, applying Theorem 5.2 again,

$$
\left\|F-F_{K}\right\|_{X \downarrow}=\lim _{N \rightarrow \infty}\left\|I_{N}\right\|_{X \downarrow} \leq \sum_{n=K+1}^{\infty}\left\|f_{n}\right\|_{X \downarrow}
$$

and so $\left\|F-F_{K}\right\|_{X \downarrow}$ tends to zero as $K \rightarrow \infty$. That is, $F_{K} \rightarrow F$ in $X \downarrow$ as $K \rightarrow \infty$. This completes the proof. 
Although $X \downarrow$ is a Banach space, it is not a Banach function space in general as the following example shows: Take $\lambda$ to be Lebesgue measure on the half line. We show that condition [1, Definition I.1.1(P5)] fails for the space $L_{\lambda \downarrow}^{2} \downarrow$. To do this we exhibit a set $E$ of finite measure and a sequence of functions $\left\{f_{n}\right\}$ in $L_{\lambda \downarrow}^{2} \downarrow$ such that $\int_{E} f_{n} /\left\|f_{n}\right\|_{L_{\lambda}^{2} \downarrow}$ is unbounded. Set

$$
E=\bigcup_{n=1}^{\infty}\left[n-2^{-n}, n\right]
$$

and note that $\lambda(E)=\sum_{n=1}^{\infty} 2^{-n}<\infty$. If $f_{n}=2^{n} \chi_{\left[n-2^{-n}, n\right]}$ we compute $\int_{E} f_{n}=1$ and $f_{n}^{\circ}=(1 / n) \chi_{[0, n]}$. Thus $\left\|f_{n}\right\|_{L_{\lambda}^{2} \downarrow}=\left\|f_{n}^{\circ}\right\|_{L_{\lambda}^{2}}=n^{-1 / 2}$ and so $\int_{E} f_{n} /\left\|f_{n}\right\|_{L_{\lambda}^{2} \downarrow}$ is unbounded for large $n$.

For the remainder of this section we investigate the dual space of $X \downarrow$.

Definition 5.4. Suppose that $g$ is a $\lambda$-measurable function. Define $\bar{g}$ by $\bar{g}(x)=\operatorname{ess}_{\sup _{t \geq x}}|g(t)|$, set $\|g\|_{X \downarrow^{\prime}}=\|\bar{g}\|_{X^{\prime}}$, and let $X \downarrow^{\prime}$ be the collection of functions $g$ for which $\|g\|_{X \downarrow^{\prime}}<\infty$.

Note that $\bar{g}$ is non-negative and non-increasing and that, by a standard measure theory argument, $\bar{g} \geq|g| \lambda$-almost everywhere. The space $X \downarrow^{\prime}$ is a subspace of $X^{\prime}$ since we have $\|g\|_{X^{\prime}} \leq\|g\|_{X \downarrow^{\prime}}$. It is easy to see that $\|\cdot\|_{X \downarrow^{\prime}}$ is a norm.

Although the notation $X \downarrow^{\prime}$ suggests the associate space of $X \downarrow$ this is not asserted here. In fact, since $X \downarrow$ is not necessarily a Banach function space, it is not clear that it has a well-defined associate space. The space $X \downarrow^{\prime}$ does behave like an associate space, however, as we see in Theorems 5.6 and 5.7 below. Theorem 5.8 shows that the dual space of $X \downarrow$ often coincides with $X \downarrow^{\prime}$. To prepare for these three theorems we need another result from $[\mathbf{1 3}]$.

Proposition 5.5. Suppose $\lambda$ satisfies (2.1), $\alpha \in(0,1)$, and $f, g$ are $\lambda$-measurable functions such that $f^{\circ}$ and $\bar{g}$ are finite $\lambda$-almost everywhere. Then there exists a non-negative $\lambda$-measurable function $h$ such that

$$
\int_{\mathbf{R}} h|g| d \lambda \geq \alpha^{2} \int_{\mathbf{R}}|f| \bar{g} d \lambda \text { and } \int_{\mathbf{R}} h \varphi d \lambda \leq \int_{\mathbf{R}}|f| \varphi d \lambda
$$

for all non-negative, non-increasing, $\lambda$-measurable functions $\varphi$.

Proof: This is proved in [13, Lemma 6.5] under the assumption that $f^{\circ} \in L_{\lambda}^{p}$ and $\bar{g} \in L_{\lambda}^{p^{\prime}}$ for some $p \in(1, \infty]$. Only the weaker assumption that $f^{\circ}$ and $\bar{g}$ are finite $\lambda$-almost everywhere is used in the proof. It remains valid in this more general situation without alteration. 
Theorem 5.6. Suppose $\lambda$ satisfies (2.1), $X$ is a rearrangement invariant space over $(\mathbf{R}, \lambda)$, and $f \in X \downarrow$. Then

$$
\|f\|_{X \downarrow}=\sup \left\{\left|\int_{\mathbf{R}} f g d \lambda\right|:\|g\|_{X \downarrow^{\prime}} \leq 1\right\} .
$$

Proof: Since $\bar{g}$ is non-increasing and $\bar{g} \geq|g| \lambda$-almost everywhere we have

$$
\begin{aligned}
\left|\int_{\mathbf{R}} f g d \lambda\right| \leq \int_{\mathbf{R}}|f||g| d \lambda \leq \int_{\mathbf{R}}|f| \bar{g} d \lambda \leq \int_{\mathbf{R}} f^{\circ} \bar{g} d \lambda \\
\leq\left\|f^{\circ}\right\|_{X}\|\bar{g}\|_{X^{\prime}}=\|f\|_{X \downarrow}\|g\|_{X \downarrow^{\prime}} .
\end{aligned}
$$

This proves that the left side of (5.1) is no less than the right side. To prove the other inequality note that if $g$ is non-negative and nonincreasing with $\|g\|_{X^{\prime}} \leq 1$ then $\|\operatorname{sgn}(f) g\|_{X \downarrow^{\prime}} \leq 1$ and $\int_{\mathbf{R}}|f| g d \lambda=$ $\left|\int_{\mathbf{R}} f \operatorname{sgn}(f) g d \lambda\right|$ so by $(1.1)$

$$
\begin{aligned}
\|f\|_{X \downarrow} & =\sup \left\{\int_{\mathbf{R}}|f| g d \lambda: g \geq 0, g \text { non-increasing, }\|g\|_{X^{\prime}} \leq 1\right\} \\
& \leq \sup \left\{\left|\int_{\mathbf{R}} f g d \lambda\right|:\|g\|_{X \downarrow^{\prime}} \leq 1\right\} .
\end{aligned}
$$

This completes the proof.

Theorem 5.7. Suppose $\lambda$ satisfies (2.1), $X$ is a rearrangement invariant space over $(\mathbf{R}, \lambda)$, and $g \in X \downarrow^{\prime}$. Then

$$
\|g\|_{X \downarrow^{\prime}}=\sup \left\{\left|\int_{\mathbf{R}} f g d \lambda\right|:\|f\|_{X \downarrow} \leq 1\right\} .
$$

Proof: The calculation in (5.2) shows that the left hand side of (5.3) is no less than the right hand side. To prove the other inequality we require Proposition 5.5. Fix $g \in X \downarrow^{\prime}$. Then $\bar{g} \in X$ so $\bar{g}$ is finite $\lambda$-almost everywhere. Fix $\alpha \in(0,1)$ and choose a non-negative function $f$ with $\|f\|_{X} \leq 1$ such that

$$
\|g\|_{X \downarrow^{\prime}}=\|\bar{g}\|_{X^{\prime}} \leq \frac{1}{\alpha} \int_{\mathbf{R}} f \bar{g} d \lambda .
$$

Since $\|f\|_{X} \leq 1, f \in X \subset X \downarrow$ so $f^{\circ}$ is finite $\lambda$-almost everywhere by Corollary 2.4. The function $h$ of Proposition 5.5 satisfies

$$
\|h\|_{X \downarrow}=\sup \int_{\mathbf{R}} h \varphi d \lambda \leq \sup \int_{\mathbf{R}}|f| \varphi d \lambda \leq\|f\|_{X} \leq 1
$$


where the suprema are taken over all non-negative, non-increasing functions $\varphi$ with $\|\varphi\|_{X^{\prime}} \leq 1$. Therefore

$$
\alpha^{3}\|g\|_{X \downarrow^{\prime}} \leq \alpha^{2} \int_{\mathbf{R}} f \bar{g} d \lambda \leq \int_{\mathbf{R}} h|g| d \lambda \leq \sup \left\{\left|\int_{\mathbf{R}} f g d \lambda\right|:\|f\|_{X \downarrow} \leq 1\right\} .
$$

Since this holds for all $\alpha \in(0,1)$ we may let $\alpha \rightarrow 1$ to obtain the remaining inequality in (5.3).

Definition 5.8. Suppose that $A$ is a Banach space of functions. We say the space $A$ has absolutely continuous norm provided every nonincreasing sequence of functions in $A$ which converges to zero pointwise, converges to zero in $A$.

In view of $[\mathbf{1}$, Proposition I.3.5] this definition agrees with $[\mathbf{1}$, Definition I.3.1] when $A$ is a Banach Function Space.

Theorem 5.9. Suppose $\lambda$ satisfies (2.1), $X$ is a rearrangement invariant space over $(\mathbf{R}, \lambda)$ and both $X$ and $X \downarrow$ have absolutely continuous norm. Then the dual space of $X \downarrow$ is $X \downarrow^{\prime}$. More precisely, each function $g \in X \downarrow^{\prime}$ gives rise to a continuous linear functional $L_{g}$ on $X \downarrow$ given by $L_{g}(f)=\int_{\mathbf{R}} f g d \lambda$. The norm of $L_{g}$ is $\|g\|_{X \downarrow^{\prime}}$ and every continuous linear functional on $X \downarrow$ is $L_{g}$ for some $g \in X \downarrow^{\prime}$.

Proof: By [1, Corollary I.4.3] $X^{\prime}=X^{*}$. If $g \in X \downarrow^{\prime}$ then $L_{g}$ is a clearly linear and Theorem 5.7 shows that $L_{g}$ is continuous on $X \downarrow$, having norm $\|g\|_{X \downarrow^{\prime}}$. Suppose now that $L$ is a continuous, linear functional on $X \downarrow$. We wish to show that $L=L_{g}$ for some $g \in X \downarrow^{\prime}$.

Since $X$ is a subspace of $X \downarrow$ (with $\|\cdot\|_{X \downarrow} \leq\|\cdot\|_{X}$ ) we may consider $L$ as a continuous linear functional on $X$. The hypothesis that $X^{*}=X^{\prime}$ shows that there is a function $g \in X^{\prime}$ such that $L f=\int_{\mathbf{R}} f g d \lambda$ for all $f \in X$. To complete the proof we show that $L f=\int_{\mathbf{R}} f g d \lambda$ for all $f \in X \downarrow$ and that $g \in X \downarrow^{\prime}$.

To do the first we fix $f \in X \downarrow$, set $f_{n}=\min (n, \max (-n, f)) \chi_{(-\infty, n]}$, and consider the sequence $\left\{\left|f_{n} g\right|\right\}$. This increases pointwise to $|f g|$. The Monotone Convergence Theorem yields

$$
\begin{aligned}
\int_{\mathbf{R}}|f g| d \lambda=\lim _{n \rightarrow \infty} & \int_{\mathbf{R}}\left|f_{n} g\right| d \lambda=\lim _{n \rightarrow \infty} L\left(\left|f_{n}\right| \operatorname{sgn}(g)\right) \\
& \leq\|L\|_{X \rightarrow \mathbf{R}} \lim _{n \rightarrow \infty}\left\|f_{n}\right\|_{X \downarrow} \leq\|L\|_{X \rightarrow \mathbf{R}}\|f\|_{X \downarrow}<\infty .
\end{aligned}
$$


Thus $f g \in L_{\lambda}^{1}$. Now consider $\left\{f_{n}\right\}$ as a sequence in $X \downarrow$. Since $\left\{\left|f-f_{n}\right|\right\}$ decreases to zero pointwise and $X \downarrow$ has absolutely continuous norm we see that $\left\{f_{n}\right\}$ converges to $f$ in $X \downarrow$. Since $L$ is continuous,

$$
L f=\lim _{n \rightarrow \infty} L\left(f_{n}\right)=\lim _{n \rightarrow \infty} \int_{\mathbf{R}} f_{n} g d \lambda=\int_{\mathbf{R}} f g d \lambda
$$

where the last inequality follows from the Dominated Convergence Theorem using our observation that $f g \in L_{\lambda}^{1}$.

The second task is to show that $g \in X \downarrow^{\prime}$. Set

$g_{n}(x)=\min (n,|g(x)|) \chi_{(\infty, n]}$ and note that $g_{n} \in X \downarrow^{\prime}$ and $\left\{g_{n}\right\}$ increases pointwise to $|g|$. Thus $\left\{\bar{g}_{n}\right\}$ increases pointwise to $\bar{g}$. The Fatou property of the Banach function space $X^{\prime}$ implies that

$$
\lim _{n \rightarrow \infty}\left\|g_{n}\right\|_{X \downarrow^{\prime}}=\lim _{n \rightarrow \infty}\left\|\bar{g}_{n}\right\|_{X^{\prime}}=\|\bar{g}\|_{X^{\prime}}=\|g\|_{X \downarrow^{\prime}}
$$

But

$$
\begin{aligned}
\left\|g_{n}\right\|_{X \downarrow^{\prime}} & =\sup \left|\int_{\mathbf{R}} f \bar{g}_{n} d \lambda\right| \\
& \leq \sup \int_{\mathbf{R}}|f||g| d \lambda \\
& =\sup L(|f| \operatorname{sgn}(g)) \leq\|L\|_{X \downarrow \rightarrow \mathbf{R}} .
\end{aligned}
$$

Here the suprema are taken over all functions $f$ with $\|f\|_{X \downarrow} \leq 1$. The conclusion is that $\|g\|_{X \downarrow^{\prime}} \leq\|L\|_{X \downarrow \rightarrow \mathbf{R}}$ so that $g \in X \downarrow^{\prime}$ as required.

Corollary 5.10. If $\lambda$ satisfies (2.1), $X$ is a rearrangement invariant space over $(\mathbf{R}, \lambda)$ and both $X$ and $X \downarrow$ have absolutely continuous norm then $X \downarrow^{\prime}$ is complete.

Proof: The dual space of any normed linear space is complete.

See [13, Example 6.9] for an example to show that $X \downarrow$ need not be reflexive even when both $X$ and $X \downarrow$ have absolutely continuous norm.

It may be that if $X$ has absolutely continuous norm then so does $X \downarrow$ but we have no proof or counterexample. In very many cases, however, it is true. We leave the following as a (non-trivial) exercise: Suppose $\lambda$ satisfies $(2.1), \Lambda(x)=\int_{-\infty}^{x} d \lambda$, and $h_{M}(x)=\min (M, 1 / \Lambda(x))$ for $M>0$. If $X$ has absolutely continuous norm and $h_{M} \in X^{\prime}$ for all $M>0$ then $X \downarrow$ has absolutely continuous norm. 


\section{References}

[1] C. Bennett And R. Sharpley, "Interpolation of operators", Academic Press Inc., Boston, MA, 1988.

[2] D. W. Boyd, Indices of function spaces and their relationship to interpolation, Canad. J. Math. 21 (1969), 1245-1254.

[3] D. W. Boyd, Indices for the Orlicz spaces, Pacific J. Math. 38 (1971), 315-323.

[4] A. Fiorenza AND M. Krbec, Indices of Orlicz spaces and some applications, Comment. Math. Univ. Carolin. 38(3) (1997), 433-451.

[5] A. Fiorenza and M. Krbec, A formula for the Boyd indices in Orlicz spaces, Funct. Approx. Comment. Math. 26 (1998), 173-179.

[6] I. Halperin, Function spaces, Canadian J. Math. 5 (1953), 273-288.

[7] G. H. Hardy, J. E. Littlewood and G. Pólya, "Inequalities", 2nd ed., Cambridge University Press, Cambridge, 1952.

[8] H. P. Heinig And A. Kufner, Hardy operators of monotone functions and sequences in Orlicz spaces, J. London Math. Soc. (2) 53(2) (1996), 256-270.

[9] G. G. Lorentz, "Bernstein polynomials", Mathematical Expositions 8, University of Toronto Press, Toronto, 1953,

[10] L. Maligranda, Indices and interpolation, Dissertationes Math. (Rozprawy Mat.) 234 (1985), 49p.

[11] M. M. RAO AND Z. D. Ren, "Theory of Orlicz spaces", Monographs and Textbooks in Pure and Applied Mathematics 146, Marcel Dekker Inc., New York, 1991.

[12] E. SAWYER, Boundedness of classical operators on classical Lorentz spaces, Studia Math. 96(2) (1990), 145-158.

[13] G. Sinnamon, Spaces defined by the level function and their duals, Studia Math. 111(1) (1994), 19-52.

Department of Mathematics

University of Western Ontario

London, Ontario, N6A 5B7

Canada

E-mail address: sinnamon@uwo.ca

Primera versió rebuda el 17 de maig de 2000, darrera versió rebuda el 10 de juliol de 2000. 